\title{
Interleukin-33 and soluble suppression of tumorigenicity 2 in scleroderma cardiac involvement
}

Francesco lannazzo

Sapienza University of Rome

Chiara Pellicano

Sapienza University of Rome

Amalia Colalillo

Sapienza University of Rome

Cesarina Ramaccini

Sapienza University of Rome

Antonella Romaniello

Sant'Andrea Hospital

Antonietta Gigante

Sapienza University of Rome

Edoardo Rosato ( $\nabla$ edoardo.rosato@uniroma1.it )

Sapienza University of Rome

\section{Research Article}

Keywords: systemic sclerosis, echocardiography, heart rate variability, IL-33, sST2

Posted Date: February 28th, 2022

DOI: https://doi.org/10.21203/rs.3.rs-1391591/v1

License: (c) (i) This work is licensed under a Creative Commons Attribution 4.0 International License. Read Full License 


\section{Abstract}

Introduction: Interleukin (IL)-33 is part of the IL-1 family of cytokines and soluble suppression of tumorigenicity 2 (SST2) is part of the family of IL-1 receptors. In systemic sclerosis (SSc), IL-33 and SST2 are involved in cardiac manifestations such as diastolic dysfunction (DD), autonomic dysfunction (AD) and right ventricular-pulmonary arterial coupling assessed by tricuspid annular plane systolic excursion (TAPSE)/systolic pulmonary artery pressure (SPAP).

Methods: Serum levels of IL33 and SST2 were assessed in 50 SSc patients and 14 healthy controls (HC). Clinical assessment, echocardiography and heart rate variability (HRV) analysis were performed in SSc patients.

Results: Serum levels of IL-33 and SST2 were significantly higher in SSc patients than HC. A linear positive correlation between modified Rodnan skin score and IL33 was observed. Serum values of sST2 were higher in SSc patients with DD than in patients without DD [15403 pg/ml (12208-19941) vs 8556 $\mathrm{pg} / \mathrm{ml}$ (6820-11036), $\mathrm{p}<0.001]$. SST2 showed a negative correlation with standard deviation of normal-tonormal RR intervals (SDNN) $(r=-0,281, p<0.05)$ and positive correlation with low frequency/high frequency $(\mathrm{LF} / \mathrm{HF})(\mathrm{r}=0,349, \mathrm{p}<0.01)$. Negative linear correlation exists between sST2 and TAPSE/sPAP $(r=-0.398$, $\mathrm{p}<0.01)$.

Conclusion: Serum levels of IL-33 and SST2 are higher in SSc patients than HC. Serum levels of SST2 are a potential marker of $\mathrm{DD}, \mathrm{AD}$ and right ventricular-pulmonary arterial coupling.

\section{Introduction}

Systemic Sclerosis (SSc) is an autoimmune disease characterized by microvascular alterations, activation of the immune system and fibrosis of skin and internal organs.

SSc can cause a wide variety of cardiac abnormalities, including microvascular coronary artery disease with cardiac Raynaud's phenomenon (RP), myocardial fibrosis, left ventricular (LV) systolic dysfunction, LV diastolic dysfunction, pericardial disease and conduction abnormalities [1]. Scleroderma heart disease is clinically manifest in approximately $10-30 \%$ of patients, although subclinical damage may be found in up to $70 \%$ of patients [2]. Diastolic dysfunction (DD) is clinically important and is a marker of increased risk of death in SSc patients [3]. The prevalence of LV systolic dysfunction (3-5\%) is lower than DD.

Most studies used conventional echocardiography to evaluate LV and right ventricular (RV) systolic and diastolic function. Recent technological advances have made pulsed TDI widely available, enabling the online measurements of velocities during a routine echographic examination to assess longitudinal LV and RV function. DD assessment includes 4 measures: left atrial volume index (LAVI), septal and lateral early relaxation velocity on tissue Doppler ( $\left.\mathrm{e}^{\prime}\right)$, early filling velocity on transmitral Doppler/early relaxation velocity on tissue Doppler (E/e') ratio, tricuspid regurgitation (TR) systolic jet velocity [4]. Recently, 
tricuspid annular plane systolic excursion (TAPSE)/systolic pulmonary artery pressure (SPAP) ratio was used as a coupling index between right ventricle and pulmonary artery [5].

Many studies have documented the presence of autonomic dysfunction (AD) in patients with SSc. AD is detected by heart rate variability (HRV) analysis. Standard deviation of normal-to-normal RR intervals (SDNN) and low frequency/high frequency (LF/HF) ratio are the most used parameters to assess AD [6]. $A D$ is an early feature of $S S c$ and it could be responsible for the reduced myocardial vasodilatory response after cold test [7]. In SSc patients with AD, intrarenal arterial stiffness, assessed by color Doppler ultrasound, is increased and exercise capacity, assessed by cardiopulmonary test, is reduced [810].

Interleukin (IL)-33 is part of the IL-1 family of cytokines and soluble suppression of tumorigenicity 2 (sST2) is part of the family of IL-1 receptors. Transcription of the ST2 receptor gene produces four isoforms of which 2 are the most important: a transmembrane receptor (ST2L or IL1RL1-b) and a soluble receptor that can be detected circulating in the serum (SST2 or IL1RL1-a). The interaction between IL-33 and ST2L activates a cascade that includes nuclear factor-KB (NF-KB), the result of which final is the production of Th2-dependent pro-inflammatory cytokines (such as IL-4, IL-5 and IL-13). SST2 greedily binds to IL-33 thus blocking the interaction between IL-33/ST2L, resulting in the abrogation of their cellular functions, thus functioning as a decoy receptor. IL-33 plays a key role in the initiation and progression of numerous fibrotic and/or inflammatory diseases such as pulmonary fibrosis, asthma, rheumatoid arthritis and inflammatory bowel diseases. [11] The interaction between IL33 and ST2L has been shown to be cardioprotective in experimental models by reducing myocardial fibrosis, preventing cardiomyocyte hypertrophy and reducing apoptosis with consequent improvement in myocardial function [12]. SST2 at the cardiac level acts in the opposite way with respect to the signal given by IL-33/ST2. sST2 demonstrated a higher predictive value of mortality than NT-proBNP in patients with acute heart failure, chronic heart failure and myocardial infarction [13].

Aim of the study is to evaluate the serum level of IL-33 and ST2 and to assess the relationship between serum level of IL-33 or ST2 and diastolic dysfunction, autonomic dysfunction and right ventricle impairment in SSc patients.

\section{Materials And Methods}

\section{Subjects}

We enrolled 50 patients [ 45 females; median age 57 (IQR 51-63) years] with diagnosis of SSc according to classification criteria of systemic sclerosis by American College of Reumatology/European Leage Against Rheumatism [14]. 29 patients had limited cutaneous SSc (IcSSc) and 21 diffuse cutaneous (dcSSc) SSc according to LeRoy et al [15].

Exclusion criteria were cardiac diseases not related to SSc, atrial fibrillation, arterial hypertension, diabetes, pulmonary diseases not related to SSc, pregnancy and breastfeeding. We also excluded SSC 
patients treated with Angiotensin converting enzyme (ACE) inhibitors, beta blockers and anti-arrhythmic drugs, angiotensin II receptor blockers, mineralocorticoid receptor antagonists.

14 healthy controls (HC) were also enrolled [12 females; median age 52 (IQR 47-65) years].

The subjects' written consents were obtained according to the Declaration of Helsinki and the study was conducted in agreement to local ethics committee directives.

\section{Serum level of IL33 and SST2}

Serum level of IL33/ (pg/ml) were measured by commercial ELISA Kit (MyBioSource, San Diego, USA). Serum level of sST2 $(\mathrm{pg} / \mathrm{ml})$ were measured by commercial ELISA kit (Abcam, Cambridge, UK). Blood samples were collected after a 12-hour overnight fast.

\section{Echocardiography}

Echocardiograms were performed with the General Electric Vivid S5 apparatus (GE Medical Systems, Israel Ltd.) All the patients were studied in left lateral decubitus. DD was evaluated in according of international guidelines of 2016 [4]. Left ventricle diameters, wall thickness, ejection fraction, right ventricle diameter, tricuspidal annular plane systolic excursion, left and right atrium area and volume, SPAP were measured.

\section{HRV}

All subjects underwent 24 hours ambulatory 3-channel ECG Holter recording (Lifecard CF, Spacelabs Healthcare, Snoqualmie,WA, USA). Autonomic nervous activity was assessed by HRV in time and frequency domain, according to the recommendation of the European Society of Cardiology and the North American Society of Pacing and Electrophysiology [16].

Spectral estimates of normal-to-normal RR intervals (NN) were obtained from stationary intervals free of ectopic beats and technical artifacts. The following parameters were computed in time domain analysis: SDNN which captures total HRV and reflects circadian heart rhythm, and the square root of the mean of the sum of the squares of differences between adjacent NN intervals (RMSSD), which correlates with the parasympathetic modulation of HR In the frequency domain Fast Fourier Transform was used to obtain power spectral estimates of HRV. Total power in the frequency range $(0-0.40 \mathrm{~Hz})$ was divided into low frequency (LF: 0.04-0.15 Hz, modulated mainly by sympathetic system) and high frequency (HF: 0.15$0.40 \mathrm{~Hz}$, modulated by parasympathetic system). HF is an indicator of parasympathetic activity; conversely LF is an indicator of sympathetic activity. Data analyses were performed with Cardionavigator plus software package (Spacelabs Healthcare, Snoqualmie, WA, USA).

\section{Clinical assessment}

Modified Rodnan skin score (mRss), disease activity index (DAl) and disease severity scale (DSS) were performed in SSc patients. Nailfold videocapillaroscopy (NVC) was performed with a videocapillaroscope 
(Pinnacle Studio Version 8) equipped with a 500x optical probe. According to Cutolo et al [17] patterns identified within the "SSc pattern" include: early, active and late.

\section{Statistical analysis}

All results are expressed as mean \pm SD or median and IQR, as appropriate. SPSS version 25.0 software was used for the statistical analysis. The Shapiro-Wilk test was used to evaluate normal distribution of data. Group comparisons were made by Student's unpaired 2-tailed t-test or Mann-Whitney test, as appropriate. Pearson product-moment correlation coefficient or Spearman's rank correlation coefficient, as appropriate, were used to test for an association between numerical variables. The chi-square test or Fisher's exact test, as appropriate, were used to compare categorical variables. Multiple regression analysis was used to evaluate the correlation between dependent variable (DD, SDNN, LF/HF and TAPSE/sPAP) and continuous independent variables. P-values $<0,05$ were considered significant.

\section{Results}

Table 1 shows epidemiological and clinical data of 50 SSc patients.

Table 1 Demographic and clinical features of systemic sclerosis patients 
Age, years - median (IQR)

Female gender - $\mathrm{n}(\%)$

Disease duration, years - median (IQR)

IcSSc/dcSSc, $n$

SSc-specific autoantibodies, n (\%)

Scl70

$22(44)$

Anticentromere

$21(42)$

Anti-RNApolimerase II

2 (4)

None

$5(10)$

\section{Nailfold video capillaroscopy, n (\%)}

Early

Active

$14(28)$

Late

$28(56)$

Ulcers history, n (\%)

mRss, median (IQR)

DAl, median (IQR)

DSS, median (IQR)

Dyastolic dysfunction, n (\%)
$27(50)$

$11(8-20)$

$1.50(0.92-3.75)$

$4(3-6)$

$8(16)$

IQR: interquartile range; ICSSC: limited cutaneous systemic sclerosis; $d c S S C$ : diffuse cutaneous systemic sclerosis; Scl70: Antitopoisomerase I antibodies; mRss. modified Rodnan skin score, DAl. disease activity index, DSS. disease severity scale.

\section{Serum level of IL-33 and SST2}

Serum levels of IL-33 were significantly higher in SSc patients than HC $[98 \mathrm{pg} / \mathrm{ml}(70-152) \mathrm{vs} 55 \mathrm{pg} / \mathrm{ml}$ (28-92), $p<0.01]$. Serum values of SST2 were significantly higher in SSc patients than $\mathrm{HC}[9115 \mathrm{pg} / \mathrm{ml}$ (6854-12696) vs 7031 (4862-8269), $p<0.05]$. In SSc patients, no correlation between serum levels of IL33 and SST2 was observed. A linear positive correlation between mRSS and IL33 $(r=0,270, p<0.05)$ was observed, where not correlation was present between mRss and SST2. IL-33 and SST2 show no correlation with disease features (DAl, DSS, disease duration).

Diastolic dysfunction 
DD was present in 8 (16\%) SSc patients. Serum values of SST2 were higher in SSc patients with DD than in patients without DD [15403 pg/ml (12208-19941) vs $8556 \mathrm{pg} / \mathrm{ml}(6820-11036), \mathrm{p}<0.001]$ (Fig. 1A). No significant differences of serum level of IL-33 were observed in SSc patients with or without DD [116 $\mathrm{pg} / \mathrm{ml}(61-167)$ vs $98 \mathrm{pg} / \mathrm{ml}$ (70-138), p >0.05] (Fig. 1B). DAl score [4 (1.84-4.46) vs 1.5 (0.84-2.59), $\mathrm{p}$ $<0.01$ ] and DSS score [6 (5-9) vs $4(3-6), p<0.05$ ] were higher in SSc with DD than SSc without DD. No significant differences of $\mathrm{mRss}$, age, disease duration were observed in SSc patients with or without DD (Table 2).

Table 2

Difference of serological markers and clinical features in systemic sclerosis patients with or without diastolic dysfunction

\begin{tabular}{|llll|}
\hline & Diastolic disfunction & No diastolic dysfunction & p value \\
\hline SST2, $(\mathrm{pg} / \mathrm{ml})$ & $15403 \mathrm{pg} / \mathrm{ml}(12208-19941)$ & $8556 \mathrm{pg} / \mathrm{ml}(6720-11224)$ & $\mathrm{p}<0.001$ \\
\hline IL-33, $(\mathrm{pg} / \mathrm{ml})$ & $116 \mathrm{pg} / \mathrm{ml}(61-167)$ & $98 \mathrm{pg} / \mathrm{ml}(70-138)$ & $\mathrm{p}>0.05$ \\
\hline SDNN & $119 \mathrm{msec}(107-127)$ & $131 \mathrm{msec}(120-143)$ & $\mathrm{p}<0.05$ \\
\hline RMSSD & $29 \mathrm{msec}(28-38)$ & $37 \mathrm{msec}(32-45)$ & $\mathrm{p}>0.05$ \\
\hline LF & $680(305-2189)$ & $751(511-1317)$ & $\mathrm{p}>0.05$ \\
\hline HF & $374(299-3164)$ & $467(299-890)$ & $\mathrm{p}>0.05$ \\
\hline LF/HF & $2.51(2.49-2.65)$ & $2.3(2.13-2.45)$ & $\mathrm{p}<0.05$ \\
\hline DAl & $4(1.84-4.46)$ & $1.5(0.84-2.59)$ & $\mathrm{p}<0.01$ \\
\hline DSS & $6(5-9)$ & $4(3-6)$ & $\mathrm{p}<0.05$ \\
\hline mRss & $11(7-18)$ & $12(8-20)$ & $\mathrm{p}>0.05$ \\
\hline $\begin{array}{l}\text { Disease duration, years } \\
\text { Age, years }\end{array}$ & $19(9-23)$ & $11(5-16)$ & $\mathrm{p}>0.05$ \\
\hline $\begin{array}{l}\text { IL-33: Interleukin-33; sST2: soluble suppression of tumorigenicity 2; SDNN: standard deviation of } \\
\text { normal-to-normal RR intervals; } R M S S D \text { : mean of the sum of the squares of differences between } \\
\text { adjacent NN intervals; } L F: \text { low frequency; HF: high frequency; } L F / H F: \text { low frequency/high frequency } \\
\text { ratio; } D A \text { : disease activity index; } D S S \text {. disease severity scale; } m R S S \text { : modified Rodnan skin score. }\end{array}$ \\
\hline
\end{tabular}

\section{HRV analysis}

Median value of SDNN is 127.8 (119.4-140) and median value of LF/HF is $2.32(2.18-2.50)$. SDNN showed negative correlation with sST2 serum level $(r=-0,281, p<0.05)($ Fig. 2A), DAl $(r=-0.307, p<0.05)$ and DSS $(r=-0.290, p<0.05)$. No correlation was observed between SDNN and IL-33, mRss, age and disease duration. In multiple regression analysis, SDNN showed a correlation with sST2 ( $\beta$ coefficient $0.306, p<0.05)$, conversely no correlation was present between SDNN and DAI ( $\beta$ coefficient $-0.172, p>$ $0.05)$ and DSS ( $\beta$ coefficient $-0.247, p>0.05)$. 
LF/HF showed a positive correlation with sST2 ( $r=0,349, p<0.01)$ (Fig. 2B). No correlation was observed between LF/HF and IL-33, mRss, DAl, DSS, age and disease duration.

SDNN values were significantly lower in patients with DD than without DD [119 msec (107-127) vs 129 msec (120-143), $p<0.05$ ], conversely LF/HF values were higher in patients with $D D$ than patients without DD [2.51 (2.49-2.65) vs $2.3(2.13-2.43), \mathrm{p}<0.05]$ (Table 2).

\section{Right ventricular function}

Median value of TAPSE/sPAP ratio was 0.78 (IQR 0.65-0.90). Negative linear correlation exists between TAPSE/sPAP and sST2 ( $r=-0.398, p<0.01)$ (Fig. 2C), DAl $(r=-0.321, p<0.05)$ and DSS $(r=-0.349, p<0.01)$. No correlation was observed between TAPSE/sPAP and IL-33, mRss, SDNN and LF/HF.

In multiple regression analysis, TAPSE/sPAP ratio showed a significant correlation with SST2 [ $\beta$ coefficiente $-0.316, p<0.05$ ], age [ $\beta$ coefficient $-0.329, p<0.01]$ and DSS [ $\beta$ coefficient $-0.311, p<0.05$ ] No correlation was present between TAPSE/sPAP ratio and DAI.

\section{Discussion}

In this study, serum levels of IL-33 and SST2 are higher in SSc patients than HC. Serum level of IL-33 shows a positive correlation with mRss, conversely serum level of sST2 does not show correlation with skin fibrosis. In SSc patients with DD, we observed higher value of serum SST2, DAI and DSS than SSc patients without DD. We not observed significant difference of serum level of IL-33 between SSc patients with or without DD. SDNN showed negative correlation with SST2 serum level and positive correlation with LF/HF. In multiple regression analysis, only SST2 showed a correlation with SDNN. Negative linear correlation exists between TAPSE/sPAP and sST2, conversely no correlation exists between TAPSE/sPAP and IL-33.

Our results confirm that IL-33 is increased in SSc patients and it shows a correlation with skin fibrosis, as assessed by mRss. Previous studies demonstrated that IL-33 is an emerging pro-fibrotic cytokine in the immune system as well as a novel potential target for systemic sclerosis [18]. While IL-33 in SSc fibrosis of lung and skin was well studied, the role of soluble ST2 (SST2) in the pathological processes and its contribution to vascular fibrosis in SSc has not been investigated. Our study demonstrated that serum sST2 is higher in SSc patients than HC, but it does not show correlation with mRss. Wagner et al demonstrated that SST2 is higher in IcSSc after 9 years of disease and SST2 serum levels were lowered by prostacyclin treatment. Serum SST2 is a biomarker for progressive vascular fibrosis [19]. Serum SST2 levels in patients with progressive disease were significantly elevated compared with patients with stable disease [20]. We can suppose that IL-33 play a key role in skin fibrosis, conversely serum level of sST2 is not involved in skin fibrosis.

In our cohort of patients, DD is present in $8(16 \%)$ patients. It is well known that DD is the consequence of myocardial fibrosis. Serum level of SST2 was significantly higher in SSc patients with DD than in SSc patients without DD, conversely IL-33 does not show significant differences in SSc patients with and without DD. Studies about SST2 are prevalently focused on myocardial infarction and heart failure. The 
role of IL-33 and SST2 in myocardial damage is not completely clear, but it is hypothesized that IL-33 is released after hearth injury. IL-33 binds to the transmembrane receptor ST2L and actives cardioprotective processes (anti-fibrotic, anti-remodeling, anti-apoptotic) [21]. SST2 enhances myocardial hypertrophy and fibrosis by blocking the favorable influence of IL-33. Its prognostic value has been reported both in acute and chronic heart failure. Since natriuretic peptides were successfully integrated into the clinical practice of heart failure, very few have made the difficult translation from initial promise to clinical application. These markers mirror the complex pathophysiology of heart failure at various levels: cell loss (troponin), fibrosis (sST2 and galectin-3), infection (procalcitonin) [22]. In systemic lupus erythematosus patients, SST2 is a marker of disease activity and subclinical DD, independent of traditional cardiovascular risk factors [23]. We can suppose that SSc patients with high serum level of IL-33 and SST2 represent a phenotype of patients with high risk of cardiac fibrosis and DD.

In this study, we demonstrated that SDNN showed a negative correlation with SST2 serum level and positive correlation with LF/HF. We did not observe any correlation between IL-33 and SDNN or LF/HF. $H R V$ is an index of autonomic activity and SDNN with LF/HF ratio are the most used parameters to assess AD.

$A D$ is an early feature of SSc and it could be responsible for the reduced myocardial vasodilatory response after cold test comparable to cardiac Raynaud's phenomenon [7]. Repeated episodes of cardiac Raynaud's phenomenon are responsible of damage of the cardiac autonomic nervous system with AD. Afterwards, cardiac ischemic episodes lead to myocardial fibrosis with DD. In addition, results of this study demonstrated that SDNN values are lower in SSc patients with DD, conversely LF/HF values are higher in SSc patients with DD than in SSc patients without DD. In SSc the abnormal sympathovagal balance expressed by the LF/HF ratio reflects the loss of the circadian rhythm with sympathetic hyperactivity (9). We can therefore hypothesize that DD and AD are simultaneously present in SSc patients and SST2 is a biomarker both DD and AD.

Our results demonstrated that a negative linear correlation exists between TAPSE/SPAP and SST2, conversely no correlation exists between TAPSE/sPAP and IL-33. The TAPSE/sPAP ratio is the echocardiographic estimate of right ventricular-pulmonary arterial coupling measured invasively by RHC. A reduced TAPSE/sPAP ratio has been associated with poor outcomes in patients with pulmonary arterial hypertension (PAH). TAPSE/SPAP ratio is a powerful predictor of all-cause mortality in patients with PAH. In SSc patients, TAPSE/sPAP ratio can be used to further select patients requiring right heart catheterization to confirm PAH diagnosis and it showed the best correlation with ventilatory efficiency and exercise capacity [24-25]. There are no data in the literature showing the role of sST2 in right ventricular dysfunction. We can suppose that the concomitant assessment of TAPSE/sPAPs ratio and serological level of SST2 could be useful to evaluate right ventricular-pulmonary arterial coupling.

Some limitations of our study need to be acknowledged. First, the sample size is small because this study is monocentric. Circulating levels of the tested fibrosis markers may not reflect the histologically proven cardiac fibrosis. The absence of histological data does not allow to attribute to serological 
markers a precise role as biomarker of myocardial damage (DD, AD and right ventricular-pulmonary arterial coupling).

In conclusion, serum levels of IL-33 and SST2 are increased in SSc patients. Serum levels of SST2 are a potential marker of $D D, A D$ and right ventricular-pulmonary arterial coupling. Future larger studies are needed to confirm our preliminary data.

\section{Declarations}

\section{Funding}

The authors declare that no funds, grants, or other support were received during the preparation of this manuscript.

\section{Competing Interests}

The authors have no relevant financial or non-financial interests to disclose.

\section{Author Contributions}

All authors contributed to the study conception and design. Material preparation, data collection and analysis were performed by [Francesco lannazzo, Chiara Pellicano, Amalia Colalillo, Cesarina Ramaccini, Antonella Romaniello, Antonietta Gigante, Edoardo Rosato]. The first draft of the manuscript was written by [Francesco lannazzo, Amalia Colalillo, Chiara Pellicano] and all authors commented on previous versions of the manuscript. All authors read and approved the final manuscript.

\section{Ethics approval}

This study was performed in line with the principles of the Declaration of Helsinki. Approval was granted by the Ethics Committee of Sapienza University of Rome (March 3, 2021 N. 6264).

\section{Consent to participate}

Informed consent was obtained from all individual participants included in the study.

\section{References}

1. Ferri C, Giuggioli D, Sebastiani M, et al. Heart involvement and systemic sclerosis. Lupus. 2005;14:702-707. doi:10.1191/0961203305lu2204oa

2. Kahan A, Allanore Y. Primary myocardial involvement in systemic sclerosis. Rheumatology (Oxford). 2006;45 Suppl 4:iv14-iv17. doi:10.1093/rheumatology/kel312

3. Tennøe AH, Murbræch K, Andreassen JC, et al. Left Ventricular Diastolic Dysfunction Predicts Mortality in Patients With Systemic Sclerosis. J Am Coll Cardiol. 2018;72:1804-1813. 
doi:10.1016/j.jacc.2018.07.068

4. Nagueh SF, Smiseth OA, Appleton CP, et al. Recommendations for the Evaluation of Left Ventricular Diastolic Function by Echocardiography: An Update from the American Society of Echocardiography and the European Association of Cardiovascular Imaging. Eur Heart J Cardiovasc Imaging. 2016;17:1321-1360. doi:10.1093/ehjci/jew082

5. Kazimierczyk R, Kazimierczyk E, Knapp M, et al. Echocardiographic Assessment of Right VentricularArterial Coupling in Predicting Prognosis of Pulmonary Arterial Hypertension Patients. J Clin Med. 2021;10:2995. doi:10.3390/jcm10132995

6. Pham T, Lau ZJ, Chen SHA, Makowski D. Heart Rate Variability in Psychology: A Review of HRV Indices and an Analysis Tutorial. Sensors (Basel). 2021;21:3998. Published 2021 Jun 9. doi:10.3390/s21123998

7. Gigante A, Galea N, Borrazzo C, et al. Role of autonomic dysfunction in the regulation of myocardial blood flow in systemic sclerosis evaluated by cardiac magnetic resonance. Int $\mathrm{J}$ Rheum Dis. 2019;22:1029-1035. doi:10.1111/1756-185X.13569

8. Gigante A, Margiotta D, Navarini L, et al. Parasympathetic activity increases with digital microvascular damage and vascular endothelial growth factor in systemic sclerosis. Clin Exp Rheumatol. 2018;36 Suppl 113:24-27.

9. Gigante A, Rosato E, Liberatori M, et al. Autonomic dysfunction in patients with systemic sclerosis: correlation with intrarenal arterial stiffness. Int J Cardiol. 2014;177:578-580. doi:10.1016/j.ijcard.2014.08.122

10. Di Paolo M, Gigante A, Liberatori M, et al. Effects of autonomic dysfunction on exercise tolerance in systemic sclerosis patients without clinical and instrumental evidence of cardiac and pulmonary involvement. Clin Exp Rheumatol. 2018;36 Suppl 113:61-67.

11. Marvie P, Lisbonne M, L'helgoualc'h A, et al. Interleukin-33 overexpression is associated with liver fibrosis in mice and humans. J Cell Mol Med. 2010;14:1726-1739. doi:10.1111/j.15824934.2009.00801.x

12. Sanada S, Hakuno D, Higgins LJ, et al. IL-33 and ST2 comprise a critical biomechanically induced and cardioprotective signaling system. J Clin Invest. 2007;117:1538-1549. doi:10.1172/JCl30634

13. Bayes-Genis A, Zhang Y, Ky B. ST2 and patient prognosis in chronic heart failure. Am J Cardiol. 2015;115:64B-9B. doi:10.1016/j.amjcard.2015.01.043

14. van den Hoogen F, Khanna D, Fransen J, et al. 2013 classification criteria for systemic sclerosis: an American college of rheumatology/European league against rheumatism collaborative initiative. Ann Rheum Dis. 2013;72:1747-1755. doi:10.1136/annrheumdis-2013-204424

15. LeRoy EC, Medsger TA Jr. Criteria for the classification of early systemic sclerosis. J Rheumatol. 2001;28:1573-1576.

16. Heart rate variability: standards of measurement, physiological interpretation and clinical use. Task Force of the European Society of Cardiology and the North American Society of Pacing and Electrophysiology. Circulation. 1996;93:1043-1065. 
17. Cutolo M, Pizzorni C, Secchi ME, Sulli A. Capillaroscopy. Best Pract Res Clin Rheumatol. 2008;22:1093-1108. doi:10.1016/j.berh.2008.09.001

18. Manetti M, Guiducci S, Ceccarelli C, et al. Increased circulating levels of interleukin 33 in systemic sclerosis correlate with early disease stage and microvascular involvement. Ann Rheum Dis. 2011;70:1876-1878. doi:10.1136/ard.2010.148247

19. Wagner A, Köhm M, Nordin A, et al. Increased Serum Levels of the IL-33 Neutralizing SST2 in Limited Cutaneous Systemic Sclerosis. Scand J Immunol. 2015;82:269-274. doi:10.1111/sji.12317

20. Günther F, Straub RH, Hartung W, et al. Increased Serum Levels of soluble ST2 as a Predictor of Disease Progression in Systemic Sclerosis. Scand J Rheumatol. 2021;1-8. doi:10.1080/03009742.2021.1929457

21. Zhang C, Li L, Feng K, et al. 'Repair' Treg Cells in Tissue Injury. Cell Physiol Biochem. 2017;43:21552169. doi:10.1159/000484295

22. de Boer RA, Daniels LB, Maisel AS, Januzzi JL Jr. State of the Art: Newer biomarkers in heart failure. Eur J Heart Fail. 2015;17:559-569. doi:10.1002/ejhf.273

23. Chorin E, Hochstadt A, Arad U, et al. Soluble ST2 and CXCL-10 may serve as biomarkers of subclinical diastolic dysfunction in SLE and correlate with disease activity and damage. Lupus. 2020;29:1430-1437. doi:10.1177/0961203320947805

24. Colalillo A, Grimaldi MC, Vaiarello V, et al. In systemic sclerosis TAPSE/sPAP ratio can be used in addition to the DETECT algorithm for pulmonary arterial hypertension diagnosis. Rheumatology (Oxford). 2021;

keab748

. doi:10.1093/rheumatology/keab748

25. Colalillo A, Pellicano C, Romaniello A, Rosato E. In systemic sclerosis TAPSE/sPAP ratio is correlated with ventilatory efficiency and exercise capacity assessed by CPET. Clin Exp Med. 2022;10.1007/s10238-022-00804-5. doi:10.1007/s10238-022-00804-5

\section{Figures}



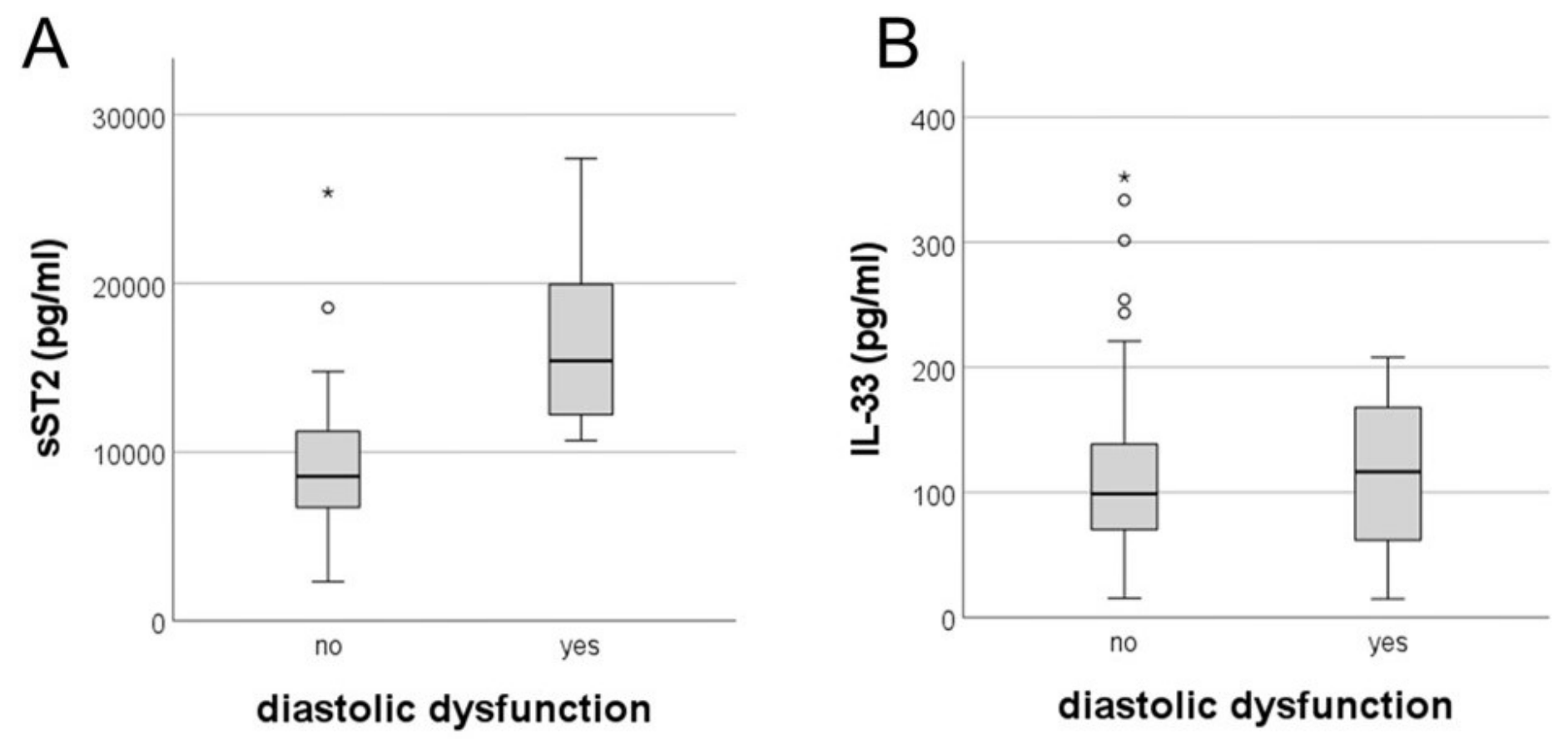

\section{Figure 1}

A Serum level of soluble suppression of tumorigenicity 2 (SST2) in systemic sclerosis patients with and without diastolic dysfunction; B Serum level of Interleukin-33 (IL-33) in systemic sclerosis patients with and without diastolic dysfunction

\section{Figure 2}

Linear correlation between sST2 and SDNN, sST2 and LF/HF, sST2 and TAPSE/sPAP 